\title{
REVIEW
}

\section{Metabolomics of human breast cancer: new approaches for tumor typing and biomarker discovery}

\author{
Carsten Denkert*, Elmar Bucher², Mika Hilvo², Reza Salek³, Matej Orešič̌2, Julian Griffin³, Scarlet Brockmöller', \\ Frederick Klauschen', Sibylle Loibl', Dinesh Kumar Barupal', Jan Budczies', Kristiina Iljin²,Valentina Nekljudova ${ }^{4}$ \\ and Oliver Fiehn ${ }^{5}$
}

\begin{abstract}
Breast cancer is the most common cancer in women worldwide, and the development of new technologies for better understanding of the molecular changes involved in breast cancer progression is essential. Metabolic changes precede overt phenotypic changes, because cellular regulation ultimately affects the use of small-molecule substrates for cell division, growth or environmental changes such as hypoxia. Differences in metabolism between normal cells and cancer cells have been identified. Because small alterations in enzyme concentrations or activities can cause large changes in overall metabolite levels, the metabolome can be regarded as the amplified output of a biological system. The metabolome coverage in human breast cancer tissues can be maximized by combining different technologies for metabolic profiling. Researchers are investigating alterations in the steady state concentrations of metabolites that reflect amplified changes in genetic control of metabolism. Metabolomic results can be used to classify breast cancer on the basis of tumor biology, to identify new prognostic and predictive markers and to discover new targets for future therapeutic interventions. Here, we examine recent results, including those from the European FP7 project METAcancer consortium, that show that integrated metabolomic analyses can provide information on the stage, subtype and grade of breast tumors and give mechanistic insights. We predict an intensified use of metabolomic screens in clinical and preclinical studies focusing on the onset and progression of tumor development.
\end{abstract}

Keywords breast cancer, metabolomics, lipidomics, biomarker analysis

*Correspondence: carsten.denkert@charite.de

'Institute of Pathology, Charité - Universitätsmedizin Berlin, 10117 Berlin, Germany Full list of author information is available at the end of the article

\section{Introduction}

Breast cancer is the most common cancer in women worldwide, with an incidence of more than 410,000 new cases per year in the USA, Europe and Japan. In OECD countries, the chance of developing invasive breast cancer in a woman's life is about 1 in 8 ( $13 \%$ of women) [1]. The disease is curable in the early stages. About $50 \%$ of patients have stage II or III tumors at the point of diagnosis and are candidates for chemo- and biological therapy. This patient group would benefit from tailored therapy that is based on biomarker testing. Although genetic alterations have been extensively characterized in breast cancer, we are just beginning to understand the changes in metabolism [2,3] that occur downstream of genomic and proteomic alterations in different types of breast tumors.

The metabolome reflects alterations in the pathophysiological state of biological systems [4]. Metabolic alterations can be the consequence of genetic changes in metabolic pathways, but they also reflect control of enzymatic activities by signaling pathways, catabolism (including membrane turnover) and competitive inhibition or activation by small molecules. Because small changes in enzyme activities can lead to large changes in metabolite levels, the metabolome can be regarded as the amplified output of a biological system [5]. Metabolomics in analogy to the terms transcriptomics and proteomics is defined as the study of all metabolites in a cell, tissue or organism for a comprehensive understanding of a biological process [6]. This is based on recently developed technologies that allow the quantitative investigation of a multitude of different metabolites. A comprehensive coverage of metabolism can be achieved only by a combination of analytical approaches. The most popular approaches for metabolomics involve gas chromatographymass spectrometry (GC-MS), liquid chromatographymass spectrometry (LC-MS) or nuclear magnetic resonance (NMR) spectroscopy. MS-based approaches are 
typically more sensitive. NMR spectroscopy can be applied to intact tissue samples and even to observe metabolites in vivo [7], with the technology being referred to as magnetic resonance spectroscopy in the clinic.

Recent metabolomics studies have improved the understanding of the basic mechanisms underlying cancer pathogenesis, which will - after translation to the clinical setting - help to improve treatment strategies. For example, phospholipids in tumor tissue are synthesized de novo; this process is increased during tumor progression [8]. This suggests that therapeutic approaches targeting lipid biosynthesis for cellular membranes might be a promising approach in breast cancer.

Here, we provide an overview of cancer metabolism, focusing on recent advances in understanding breast cancer metabolism. We examine results from the European FP7 METAcancer project, which combined the three major technologies for metabolic profiling (GCMS, LC-MS and NMR) to maximize metabolite coverage (Figure 1). This project aimed at characterizing the metabolism of breast cancer to identify new biomarkers and new targets for therapeutic interventions, and we compare these findings with results from other groups working in this area. We discuss how such data can be further evaluated by mining available databases, including expression data at the transcriptional level, as well as by additional investigations on protein and mRNA markers relevant for metabolic alterations.

\section{What do we know about cancer metabolism?}

Several recent publications have shown that metabolomics can be used to investigate changes in tumor tissue related to apoptosis, hypoxia and energy metabolism $[9,10]$. However, it is not clear how mutations in tumor cells, and specifically in metastatic tumor cells, help establish the known hallmarks of cancer cell metabolism $[11,12]$. Otto Warburg made the seminal observation [13] that glucose usage in tumors does not lead to production of excess NADH for subsequent oxidative phosphorylation in mitochondria for production of ATP, but instead lactate accumulates, even when enough oxygen is present for mitochondrial respiration. Despite this seemingly less efficient use of glycolysis, large amounts of glucose are taken up by tumor cells. Clinically, this phenomenon can be used for the detection of tumors by positron emission tomography (PET) imaging. In tumor cells glucose is not used for production of NADH and ATP, but instead biosynthetic pathways are activated that lead to accumulation of building blocks for biopolymers that sustain rapid cell growth, namely glycerol-3phosphate (via glycerol-3-phosphate acyltransferase 1 (GPAM) from triose phosphate intermediates) [14] and, more importantly, NADPH production via the pentose phosphate pathway [15]. NADPH is a key metabolite for cell division [16]. Although many studies of NADPH production have focused on its role in the regulation of redox milieus, in particular for antioxidant activity via regenerating glutathione pools [17], NADPH production can more easily be explained by the general need for reductive energy in anabolic reactions [15], specifically in fatty acid biosynthesis [18] and increased generation of polar lipids that are required for production of cellular membranes during cell division. Secondary products of the pentose phosphate pathways are pentose phosphates, which are required for production of nucleotides in DNA and RNA and thus can also indicate cell division. The increase in metabolic fluxes towards biosynthesis of lipids is also marked by accumulation of cholines, which has been observed by NMR-based metabolomics in several studies $[19,20]$. However, until recently, the scientific community had been puzzled by one remarkable discrepancy: if many classic mutations in cancer biology can be explained by fostering dysregulation of cellular metabolism in favor of production of choline, glycerol-3-phosphate and NADPH as building blocks [18] for polar membrane lipids, how is the main fatty acid biosynthetic substrate acetyl-CoA produced? When glycolysis is used in conjunction with mitochondrial oxidation of NADH, acetyl-CoA is a direct product of oxidative decarboxylation of pyruvate through the pyruvate dehydrogenase complex. However, as stated above, cancer cells produce lactate instead of pyruvate; thus, less acetyl-CoA is produced through glycolysis (Figure 2). In a recent study using metabolomics, stable isotope flux analysis demonstrated that glutamine metabolism provides acetyl-CoA in the reverse reaction of citrate synthase, using citrate lyase, which splits citric acid into oxaloacetate and acetyl-CoA [21]. Under mutation of isocitrate dehydrogenase 1 [22], flux in the Krebs cycle is partly reversed under hypoxic conditions, unlike in normoxic media [21] (Figure 2). Citrate is produced using reductive carboxylation of glutaminederived $\alpha$-ketoglutarate and, overall, much less NADH is produced in cancer cells than in non-malignant cells under aerobic conditions. These results showed that studies in cancer cell lines that were undertaken under normal oxygen levels might lead to false interpretations, and that greater care must be used to mimic in vivo tumor microenvironments in mechanistic studies targeting metabolic regulation.

One of the other profound changes that accompany tumor proliferation is alteration in the proportion of choline-containing metabolites. Choline, phosphocholine and glycerophosphocholine can be observed in clinical magnetic resonance spectroscopy. Numerous studies have noted alterations in choline metabolism during cancer cell metabolism, and these metabolites have been used for classifying tumor types [23,24] during the 


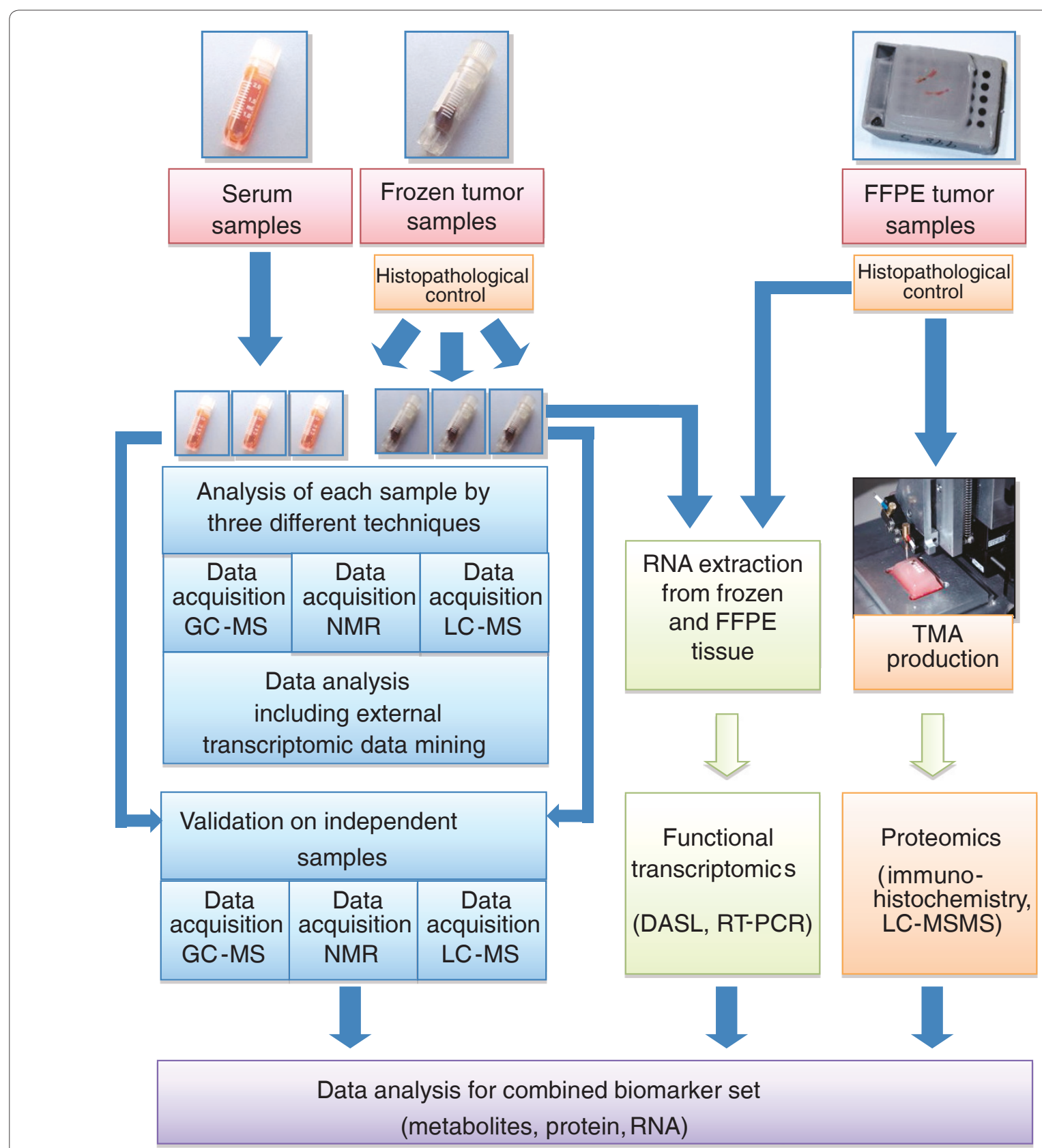

Figure 1. Workflow of samples in the METAcancer project. Tissue samples were analyzed in parallel with mass spectrometry (GC-MS and LC-MS) and nuclear magnetic resonance (NMR) spectroscopy. The metabolic profiles were linked to the analysis of mRNA markers and protein markers. DASL, CDNA-mediated annealing, selection, extension, and ligation assay; FFPE, formalin-fixed, paraffin-embedded; RT- PCR, reverse transcriptase PCR; TMA, tissue microarray.

immortalization of cell lines [25] and during apoptosis and necrosis [26,27]. These changes are thought to represent the turnover in cell membranes, with perturbations in choline metabolism reflecting either the synthesis or degradation of the cell membranes in the tumors. In addition, changes in mobile lipid droplets have been observed [28]. Such lipids move through the cytosol at a similar rate to small molecules; their tumbling nature allows detection by solution state NMR spectroscopy [29]. Mobile lipid droplets have been associated with a number of processes, including proliferation [30], inflammation [31], malignancy [32], growth arrest, necrosis [33] and apoptosis [34], using specific NMR resonances for unsaturated or saturated lipid acyl chains. Mobile lipids are thought to represent either the accumulation of lipids in cytosolic vesicles or the 
(a)

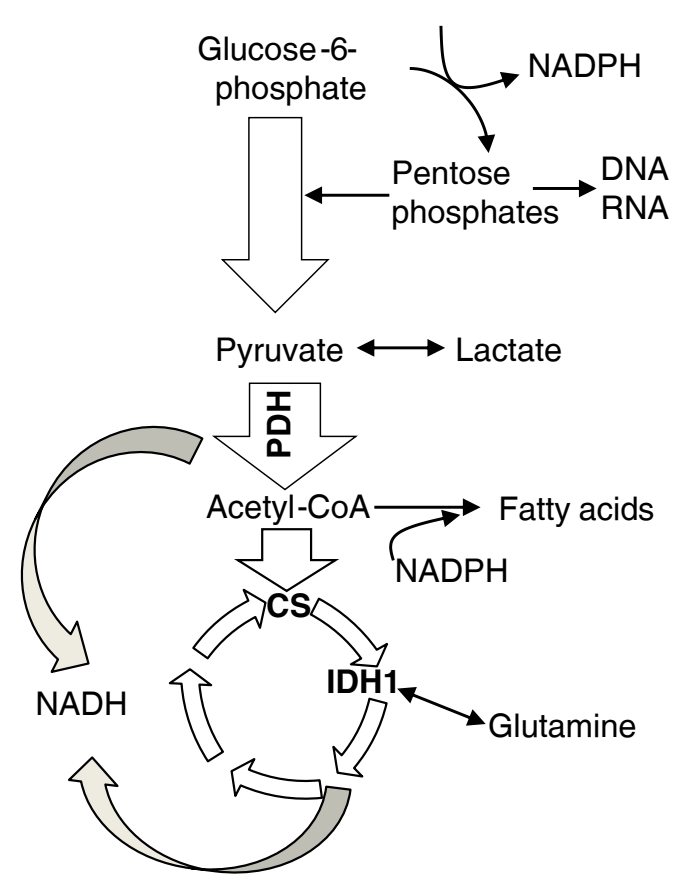

(b)

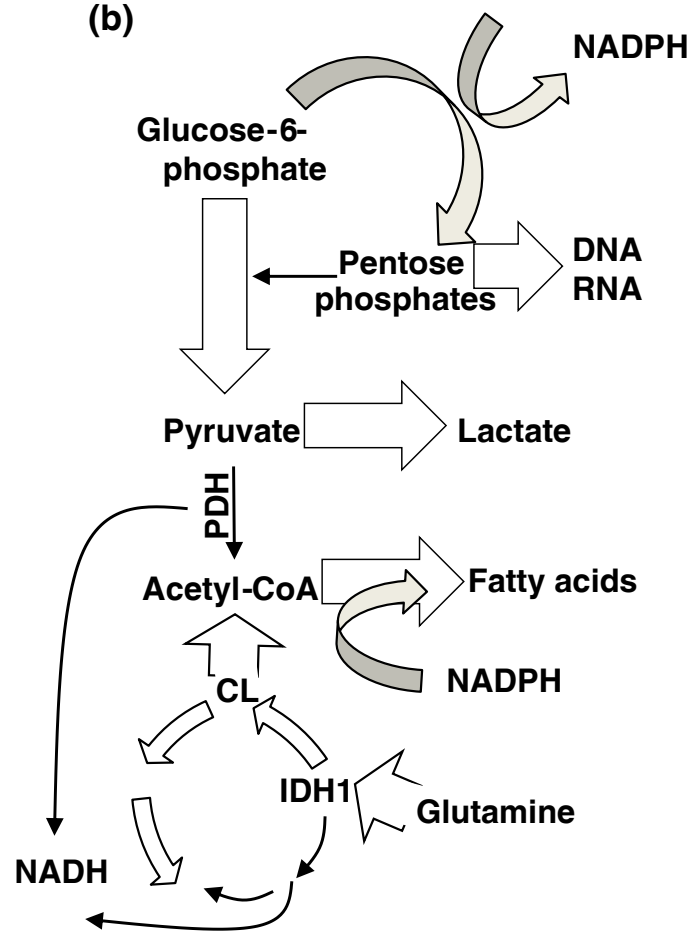

Figure 2. Simplified schema of major metabolic fluxes in (a) aerobic non-malignant cells and (b) hypoxic tumor cells of breast carcinoma. Thickness of arrows and bold text indicate relative intensity of fluxes. $\mathrm{CL}$, citrate lyase; $\mathrm{CS}$, citrate synthase; IDH1, isocitrate dehydrogenase 1; PDH, pyruvate dehydrogenase. According to Metallo et al. [21], the increased flux from glutamine into the Krebs cycle by mutation of IDH1 provides the acetyl-CoA for lipid biosynthesis under hypoxic conditions, because most pyruvate in cancer cells is converted to lactate. Increase in flux through the pentose phosphate pathway delivers ribose-5-phosphate needed for DNA synthesis and NADPH required for lipid biosynthesis. Conversely, less NADH is produced through pyruvate dehydrogenase or the Krebs cycle, as mitochondrial respiration for ATP production is less favored.

formation of microdomains in the cell membrane [35]. Furthermore, there is evidence that some of these lipid changes may be characteristic of distinct processes. A selective accumulation of polyunsaturated lipids compared with saturated lipids appears to be distinct to apoptosis rather than necrosis [36,37], for example, in glioma [28].

\section{Metabolomic approach for classification of tissue types}

The European FP7 METAcancer consortium analyzed breast tumor and non-malignant breast tissue samples from over 300 patients with three different technologies (Figure 1). In combination, more than 600 metabolites could be structurally identified, consisting of complex lipids [8], primary metabolites and a vast array of unidentified metabolic signals. Although metabolome coverage cannot be computed [38] (unlike for other genomics technologies), this number of identified metabolites is certainly the largest ever reported in human breast carcinoma. Metabolomic technologies are complementary in metabolome coverage and can be used for structural identification of hundreds of metabolites [39], many of which could be identified and linked to biochemical pathways and to evaluate changes in metabolic pathways in different types of tissue.

As expected, the largest differences were observed between normal breast tissue and malignant breast tumors [8]. With the metabolomics approach it is possible to distinguish between normal and malignant breast tissue in unsupervised and supervised analyses; an example is shown in Figure 3. Unsupervised multivariate statistics is used to display the overall data variance in large datasets, whereas supervised statistics tries to identify only those data that contributed most to a statistical question [40], such as different cancer stages. Metabolomics data from breast cancer patients can be used in a similar way to gene expression microarray data, using, for example, hierarchical clustering and heat maps. Using data generated in the METAcancer project [8], the resulting heat map (Figure 3) of 15 normal and 289 cancer tissue samples showed a clear separation of 


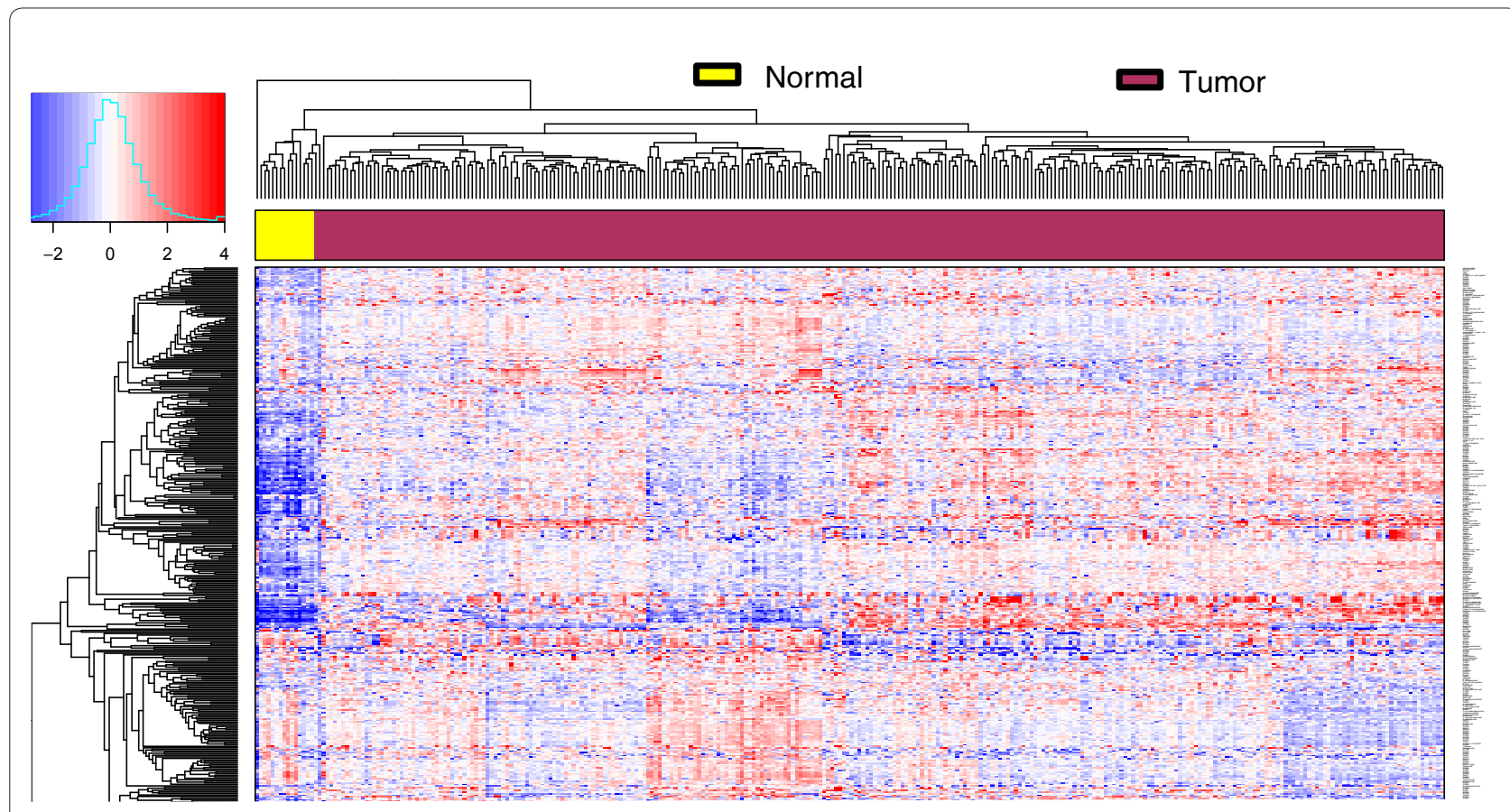

Figure 3. Heat map derived from the GCTOF MS metabolomics dataset comparing 289 tumor samples and 15 normal samples [14]. Metabolites are plotted on the $y$-axis and samples on the $x$-axis. Data were log2-transformed and median centered in a metabolite-wise manner. Blue indicates data points with a value smaller than the median of the respective metabolite and red indicates higher values. The hierarchical clustering reveals that the measured metabolites can separate normal and cancer tissues. Only two cancer samples cluster together with the normal samples, and one normal sample behaves as an outlier.

normal and cancer samples on the basis of the detected metabolites with very few outliers or cancer samples that were misclassified as non-malignant tissues. In addition, metabolic differences were observed between different types of breast cancer. The major groups of breast cancer, hormone receptor-positive and -negative tumors, have a different biological background, different clinical characteristics and are treated by different therapeutic strategies. Similarly, it was possible to detect differences between grade 1 or 2 and grade 3 tumors. Therefore, we conclude that it is possible to generate meaningful biological information from tumor tissue by metabolic profiling.

\section{Changes in membrane phospholipid synthesis are involved in the progression of breast cancer}

Analyzing the LC-MS lipidomics data, significant differences were observed in the tumor compared with normal tissue (Figure 3) [8]. In tumors, it was observed that tumor grade and estrogen receptor (ER) status affect the lipid profiles most radically. Phospholipids containing major (C16:0 fatty acids, where 16 is the number of carbon atoms and 0 is the number of double bonds) or minor (C14:0 and C18:0 fatty acids) products of the fatty acid synthase (FASN) enzyme, showed the highest changes regarding ER status and grade. The majority of the ER-negative tumors are of grade 3 [41], which was evident also in our patient population. Thus, either grade or ER status could explain these results. The ER status only within grade 3 tumors and the grade only within ERpositive tumors was therefore analyzed, confirming that both ER status and grade independently affected the same lipids, with the highest levels found in ER-negative grade 3 tumors. Increased levels of several related phospholipids were also associated with poorer overall survival of the patients [8].

On the basis of the lipidomics findings and using the GeneSapiens in silico transcription database [42] for data mining, key enzymes of lipid biosynthesis were identified (Figure 4). These enzymes were stained by immunohistochemistry in tumor tissue and analyzed together with the LC-MS data of the same patient cohort. In particular, FASN and acetyl-CoA carboxylase 1 (ACACA), which are two major enzymes involved in de novo fatty acid synthesis, were increased at the protein level in the tumors that had high levels of phospholipids containing de novo synthesized fatty acids [8]. These results show that it is possible to connect protein expression data by immunohistochemistry with LC-MS lipidomic profiles of the identical tumors.

To evaluate the function of these lipid-metabolizing enzymes for breast cancer proliferation and apoptosis, 


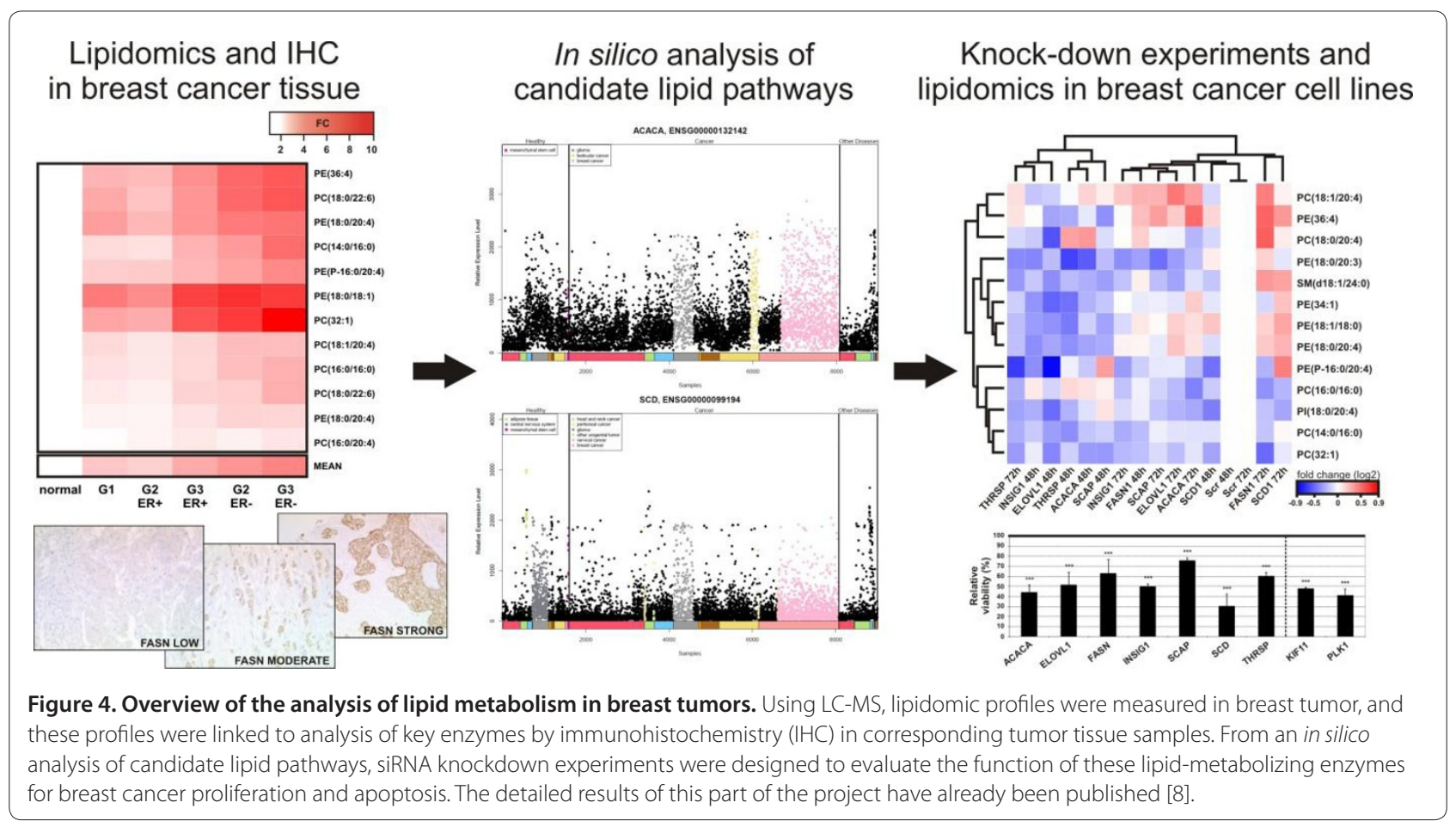

breast cancer cell lines were investigated by downregulating several central genes using small interfering RNA (siRNA) - for example, elongation of very long chain fatty acid-like 1 (ELOVL1), FASN, stearoyl-CoA desaturase $(\mathrm{SCD})$, and sterol regulatory element-binding protein cleavage-activating protein (SCAP). This resulted in decreased cell viability and lower phospholipid levels measured by LC-MS [8]. Increased de novo lipid synthesis is typically found in tumor cells but not in normal cells, which would make it a promising target for new tumorspecific therapeutic approaches.

\section{NMR-based metabolites separate ductal from lobular carcinoma, different tumor grades and different molecular subtypes}

In addition to MS-based approaches, ${ }^{1} \mathrm{H}$ high-resolution magic angle spinning (HRMAS) ${ }^{1} \mathrm{H}$ NMR spectroscopy can be used to profile tumor metabolism in intact tumors. HRMAS ${ }^{1} \mathrm{H}$ NMR spectroscopy has been applied to a wide range of intact tumors, including prostate cancer, human brain tissue, liposarcoma, malignant lymph nodes, kidney cancer and glioma [36,43-46].

Using a combination of HRMAS ${ }^{1} \mathrm{H}$ NMR spectroscopy and multivariate statistics, spectra from cancer and normal tissues are readily separated, both normal breast and ductal tissue $[47,48]$. Normal breast tissue typically does not contain many of the small-molecule metabolites, such as amino acids, glycolytic intermediates and choline-containing metabolites, found in breast tumors.
A more difficult, but more relevant, classification problem is the discrimination of different tumor types or grades. Using HRMAS ${ }^{1} \mathrm{H}$ NMR spectroscopy and multivariate statistics, invasive ductal cancer could be separated from lobular cancer [49]. Using data from the METAcancer consortium [8], tumors of grade 1 and 3 could readily be separated by NMR-based metabolomic analysis, although tumor grade 2 formed a dispersed group between these two extremes, as might be expected. The separation of ER-positive and progesteronereceptor-positive tissue by NMR was poor and only marginally better than the random predicted model from the negative samples. Giskeødegård and colleagues [50] have shown that HRMAS ${ }^{1} \mathrm{H}$ NMR spectroscopy can be used to determine ER and progesterone receptor status and lymph node status, with classifications ranging from $68 \%$ to $88 \%$ for these three status markers on a dataset of 50 tumors. At present, data from neither of these studies $[49,50]$ are validated as clinical diagnostic tools for treatment, but they do indicate that NMR-based metabolomics detects altered metabolism in different subtypes of breast tumors despite the challenges of building datasets from multiple sites and of having variable amounts of healthy breast tissue associated with biopsy samples, as in the METAcancer study.

\section{Combined markers - GPAM analysis and metabolic profiles}

GPAM is one of the key enzymes in the biosynthesis of triacylglycerols and phospholipids. Therefore, the 
expression of GPAM in malignancies is of particular interest as phospholipids are an important and major component of all cell membranes. To investigate the protein expression of GPAM, 228 breast cancer samples were immunohistochemically stained and evaluated [14]. This study showed that GPAM is differentially expressed in malignant breast tumors and that the levels of its substrate sn-glycerol-3-phosphate level are higher in breast cancer than in normal breast tissue. The immunohistochemical results showed a significant correlation of high cytoplasmic GPAM expression with negative hormone receptor status and a significant correlation of high GPAM expression with a better overall patient survival. In the metabolomic analysis, GPAM expression is associated with increased levels of phospholipids, especially phosphatidylcholines [14]. The results indicated that, in breast cancer, GPAM affects the level of phospholipids more than it does triglycerides, and most of the changes are independent of those associated with ER status and tumor grade. This implies that, at least in the context of breast cancer, the function of GPAM is directed more towards phospholipid production rather than triglyceride synthesis. Accordingly, it has been shown previously that increased de novo fatty acid synthesis is a hallmark of cancer cells and the products of this lipogenic pathway are directed mainly to the cell membrane phospholipids [8].

To extend the number of metabolic pathways covered by the analysis, an additional GC-MS-based metabolic analysis was performed. GC-MS-based analyses of metabolic impact or changes in metabolism have a long history in breast cancer research - for example, analysis of phospholipids [51], pharmacology (including tamoxifen metabolism) [52,53], exposure to xenobiotics [54], estrogen levels [55] or urinary metabolomic profiles $[56,57]$. However, clinical and biochemical phenotypes of large sets of breast cancer tissues had not been published until recently. Using retention-index-based metabolomic libraries [58], GC-MS-based metabolomics of breast cancer tissue analyses led to the identification of 467 metabolites [14]. Of these, 161 metabolites had a known chemical structure and could be mapped to metabolite names. The analysis from the GPAM expression with the data showed 57 significantly changed metabolites $(P<0.05)$. Of these 57 metabolites, 19 could be identified [14]. Pathway analysis led to a more detailed investigation of GPAM, showing significant correlation of GPAM expression with survival of patients, clinico-pathological features and metabolomic and lipidomic profiles.

The identification of differentially regulated, but structurally unidentified, metabolites in untargeted metabolomic analyses remains a major bottleneck for advancement of the field and more comprehensive understanding of cancer metabolism, including metabolic regulators. A novel approach using
GC-MS-based accurate mass spectrometry in conjunction with a cheminformatics workflow recently showed how unknown metabolites can be identified from breast cancer tissues [59]. Accurate mass spectrometry can be used to derive unique elemental compositions [60], which are then used to screen a diverse set of biochemical and chemical databases. On average, over 500 different chemical structures are retrieved in such accurate-mass-based searches [59]. The concept is to use multiple lines of evidence to constrain these hit lists - for example, by structure-based prediction of chromatographic retention times [61], by using substructure information [62], such as the number of acidic protons, and by excluding structures that do not match the detected accurate masses of ion fragmentation pathways [63]. Although this workflow method [59] is not yet available as a standalone software program, it outlines the pathway to new discoveries using advanced instrumentation and database queries.

\section{Impact for cancer research and clinical management of breast cancer patients}

Treatment options for breast cancer are initially identified according to the stage of the disease. There are several modes of treatment, including surgery, radiotherapy, endocrine therapy and cytotoxic chemotherapy. However, the management of the disease for an individual patient will depend on many factors, including menopausal status, hormone receptor status and treatment preferences. The final treatment regimen selected, following discussion with the patient, will depend on the individual circumstances, making it difficult to manage the disease by following a systematic treatment algorithm. To enable a better stratification of patients, it is important to identify the pathways that are relevant for tumor progression and therapy response and to determine biomarkers that could be used to monitor the activity of those pathways.

Metabolomic analyses by GC-MS, LC-MS and NMR spectroscopy are suitable for the analysis of tumor tissue and provide valuable information that can be integrated with proteomic and transcriptomic data. This makes it possible to study the changes in malignant tumors by constructing a correlation network that consists of combined RNA, protein and metabolite data. This will lead to new insights into the mechanisms of cancer progression and cancer patient management through improved molecular diagnostics leading to improved therapeutic concepts by selection of effective drugs as part of systems medicine. The METAcancer project has been the first large-scale effort to combine the three major metabolomic technology platforms for analyzing a major human disease. This improves the likelihood that genuine metabolic biomarkers for breast cancer tissues can be 
detected and validated, and will eventually lead to diagnostic toolkits that will facilitate a much more precise predictive and prognostic assessment. Current research efforts are focused on the integration of high quality metabolomic data to proteomics and transcriptomics data to enable better insights into the cellular mechanisms that define the onset and progression of breast carcinoma as a basis for clinical applications.

\section{Abbreviations}

ER, estrogen receptor; FASN, fatty acid synthase; GC-MS, gas chromatographymass spectrometry; GPAM, glycerol-3-phosphate acyltransferase 1; HRMAS, ${ }^{1} \mathrm{H}$ high-resolution magic angle spinning; LC-MS, liquid chromatography-mass spectrometry; NMR, nuclear magnetic resonance; PET, positron emission tomography; siRNA, small interfering RNA.

\section{Acknowledgements}

The METAcancer project was funded by the European Commission in the FP7 Framework.

\section{Conflicts of interest}

The authors declare no conflicts of interest.

\section{Author details}

'Institute of Pathology, Charité - Universitätsmedizin Berlin, 10117 Berlin, Germany. ${ }^{2}$ Biotechnology for Health and Well-being, VTT Technical Research Centre of Finland, Espoo and Turku, PO Box 1000, FI-02044 VTT, Finland. ${ }^{3}$ Department of Biochemistry, University of Cambridge, Cambridge, CB2 1GA, UK. ${ }^{4}$ German Breast Group, GBG-Forschungs GmbH, 63263 Neu-Isenburg, Germany. ${ }^{5}$ Genome Center, University of California Davis, Davis, CA 95616, USA.

Published: 30 April 2012

\section{References}

1. Siegel R, Naishadham D, Jemal A: Cancer statistics, 2012. CA Cancer J Clin 2012, 62:10-29.

2. Claudino WM, Quattrone A, Biganzoli L, Pestrin M, Bertini I, Di Leo A: Metabolomics: available results, current research projects in breast cancer, and future applications. J Clin Oncol 2007, 25:2840-2846.

3. Griffin JL: The Cinderella story of metabolic profiling: does metabolomics get to go to the functional genomics ball? Philos Trans R Soc London B Biol Sci2006, 361:147-161.

4. Fiehn O: Metabolomics - the link between genotypes and phenotypes. Plant Mol Biol 2002, 48:155-171.

5. Denkert C, Budczies J, Kind T, Weichert W, Tablack P, Sehouli J, Niesporek S, Könsgen D, Dietel M, Fiehn O: Mass spectrometry-based metabolic profiling reveals different metabolite patterns in invasive ovarian carcinomas and ovarian borderline tumors. Cancer Res 2006, 66:10795-10804

6. Fiehn O, Kopka J, Dörmann P, Altmann T, Trethewey RN, Willmitzer L: Metabolite profiling for plant functional genomics. Nat Biotechnol 2000, 18:1157-1161.

7. Bathen TF, Sitter B, Sjøbakk TE, Tessem MB, Gribbestad IS: Magnetic resonance metabolomics of intact tissue: a biotechnological tool in cancer diagnostics and treatment evaluation. Cancer Res 2010, 70:6692-6696.

8. Hilvo M, Denkert C, Lehtinen L, Müller B, Brockmöller S, Seppänen-Laakso T, Budczies J, Bucher E, Yetukuri L, Castillo S, Berg E, Nygren H, Sysi-Aho M, Griffin JL, Fiehn O, Loibl S, Richter-Ehrenstein C, Radke C, Hyötyläinen T, Kallioniemi O, Iljin K, Oresic M: Novel theranostic opportunities offered by characterization of altered membrane lipid metabolism in breast cancer progression. Cancer Res 2011, 71:3236-3245.

9. Weljie AM, Jirik FR: Hypoxia-induced metabolic shifts in cancer cells: Moving beyond the Warburg effect. Int J Biochem Cell Biol 2011, 43:981-989.

10. Griffin JL, Shockcor JP: Metabolic profiles of cancer cells. Nat Rev Cancer 2004, 4:551-561.

11. Hsu PP, Sabatini DM: Cancer cell metabolism: Warburg and beyond. Cell 2008, 134:703-707.

12. Vander Heiden MG, Cantley LC, Thompson CB: Understanding the Warburg effect: the metabolic requirements of cell proliferation. Science 2009, 324:1029-1033.

13. Warburg O: On respiratory impairment in cancer cells. Science 1956, 124:269-270.

14. Brockmöller SF, Bucher E, Müller BM, Budczies J, Hilvo M, Griffin JL, Orešič M, Kallioniemi O, Iljin K, Loibl S, Darb-Esfahani S, Sinn BV, Klauschen F, Prinzler J, Bangemann N, Ismaeel F, Fiehn O, Dietel M, Denkert C: Integration of metabolomics and expression of glycerol-3-phosphate acyltransferase (GPAM) in breast cancer-link to patient survival, hormone receptor status, and metabolic profiling. J Proteome Res 2012, 11:850-860.

15. Cairns RA, Harris IS, MakTW: Regulation of cancer cell metabolism. Nat Rev Cancer 2011, 11:85-95.

16. Vander Heiden MG, Cantley LC, Thompson CB: Understanding the Warburg effect: the metabolic requirements of cell proliferation. Science 2009, 324:1029-1033.

17. Schafer FQ, Buettner GR: Redox environment of the cell as viewed through the redox state of the glutathione disulfide/glutathione couple. Free Radical Biol Med 2001, 30:1191-1212.

18. DeBerardinis RJ, Sayed N, Ditsworth D, Thompson CB: Brick by brick: metabolism and tumor cell growth. Curr Opin Genet Dev 2008, 18:54-61.

19. Ackerstaff E, Glunde K, Bhujwalla ZM: Choline phospholipid metabolism: a target in cancer cells? J Cell Biochem 2003, 90:525-533.

20. Glunde K, Bhujwalla ZM, Ronen SM: Choline metabolism in malignant transformation. Nat Rev Cancer 2011, 11:835-848.

21. Metallo CM, Gameiro PA, Bell EL, Mattaini KR, Yang J, Hiller K, Jewell CM, Johnson ZR, Irvine DJ, Guarente L, Kelleher JK, Vander Heiden MG, lliopoulos O, Stephanopoulos G: Reductive glutamine metabolism by IDH1 mediates lipogenesis under hypoxia. Nature 2011, 481:380-384

22. Dang L, White DW, Gross S, Bennett BD, Bittinger MA, Driggers EM, Fantin VR, Jang HG, Jin S, Keenan MC: Cancer-associated IDH1 mutations produce 2-hydroxyglutarate. Nature 2009, 462:739-744

23. Gill S, Small R, Thomas D, Patel P, Porteous R, Van Bruggen N, Gadian D, Kauppinen R, Williams S: Brain metabolites as 1H NMR markers of neuronal and glial disorders. NMR Biomed 1989, 2:196-200.

24. Peeling J, Sutherland G: High-resolution 1H NMR spectroscopy studies of extracts of human cerebral neoplasms. Magn Reson Med 1992, 24:123-136.

25. Bhakoo KK, Williams SR, Florian CL, Land H, Noble MD: Immortalization and transformation are associated with specific alterations in choline metabolism. Cancer Res 1996, 56:4630-4635.

26. Griffin JL, Blenkiron C, Valonen PK, Caldas C, Kauppinen RA: High-resolution magic angle spinning $1 \mathrm{H}$ NMR spectroscopy and reverse transcriptionPCR analysis of apoptosis in a rat glioma. Anal Chem 2006, 78:1546-1552.

27. Valonen PK, Griffin JL, Lehtimäki KK, Liimatainen T, Nicholson JK, Gröhn OHJ, Kauppinen RA: High-resolution magic-angle-spinning 1H NMR spectroscopy reveals different responses in choline-containing metabolites upon gene therapy-induced programmed cell death in rat brain glioma. NMR Biomed 2005, 18:252-259.

28. Rémy C, Fouilhé N, Barba I, Sam-Laï E, Lahrech H, Cucurella MG, Izquierdo M, Moreno A, Ziegler A, Massarelli R: Evidence that mobile lipids detected in rat brain glioma by $1 \mathrm{H}$ nuclear magnetic resonance correspond to lipid droplets. Cancer Res 1997, 57:407.

29. Auer DP, Gössl C, Schirmer T, Czisch M: Improved analysis of 1H-MR spectra in the presence of mobile lipids. Magn Reson Med 2001, 46:615-618.

30. Barba I, Cabañas ME, Arús C: The relationship between nuclear magnetic resonance-visible lipids, lipid droplets, and cell proliferation in cultured C6 cells. Cancer Res 1999, 59:1861.

31. Wilken B, Dechent P, Herms J, Maxton C, Markakis E, Hanefeld F, Frahm J: Quantitative proton magnetic resonance spectroscopy of focal brain lesions. Pediatr Neurol 2000, 23:22-31.

32. Kuesel AC, Donnelly SM, Halliday W, Sutherland GR, Smith ICP: Mobile lipids and metabolic heterogeneity of brain tumours as detectable by Ex Vivo1H MR spectroscopy. NMR Biomed 1994, 7:172-180.

33. Zoula S, Herigault G, Ziegler A, Farion R, Decorps M, Remy C: Correlation between the occurrence of 1H-MRS lipid signal, necrosis and lipid droplets during C6 rat glioma development. NMR Biomed 2003, 16:199-212.

34. Liimatainen T, Hakumäki JM, Kauppinen RA, Ala-Korpela M: Monitoring of gliomas in vivo by diffusion MRI and 1H MRS during gene therapyinduced apoptosis: interrelationships between water diffusion and mobile lipids. NMR Biomed 2009, 22:272-279.

35. Luciani AM, Grande S, Palma A, Rosi A, Giovannini C, Sapora O, Viti V, Guidoni $\mathrm{L}$ : Characterization of $1 \mathrm{H}$ NMR detectable mobile lipids in cells from 
human adenocarcinomas. FEBS J 2009, 276:1333-1346.

36. Griffin JL, Lehtimäki KK, Valonen PK, Gröhn OHJ, Kettunen MI, Ylä-Herttuala S, Pitkänen A, Nicholson JK, Kauppinen RA: Assignment of $1 \mathrm{H}$ nuclear magnetic resonance visible polyunsaturated fatty acids in BT4C gliomas undergoing ganciclovir-thymidine kinase gene therapy-induced programmed cell death. Cancer Res 2003, 63:3195-31201.

37. Lehtimäki KK, Valonen PK, Griffin JL, Väisänen TH, Gröhn OHJ, Kettunen MI, Vepsäläinen J, Ylä-Herttuala S, Nicholson J, Kauppinen RA: Metabolite changes in BT4C rat gliomas undergoing ganciclovir-thymidine kinase gene therapy-induced programmed cell death as studied by $1 \mathrm{H}$ NMR spectroscopy in vivo, ex vivo, and in vitro. J Biol Chem 2003, 278:45915-45923

38. Kind T, Scholz M, Fiehn O: How large is the metabolome? A critical analysis of data exchange practices in chemistry. PLoS One 2009, 4:e5440.

39. Kind T, Fiehn O: Advances in structure elucidation of small molecules using mass spectrometry. Bioanalyt Rev 2010, 2:23-60.

40. Goodacre R, Vaidyanathan S, Dunn WB, Harrigan GG, Kell DB: Metabolomics by numbers: acquiring and understanding global metabolite data. Trends Biotechnol 2004, 22:245-252.

41. Loi S, Haibe-Kains B, Desmedt C, Lallemand F, Tutt AM, Gillet C, Ellis P, Harris A, Bergh J, Foekens JA: Definition of clinically distinct molecular subtypes in estrogen receptor-positive breast carcinomas through genomic grade. J Clin Oncol 2007, 25:1239-1246.

42. Kilpinen S, Autio R, Ojala K, lljin K, Bucher E, Sara H, Pisto T, Saarela M, Skotheim Rl, Björkman M: Systematic bioinformatic analysis of expression levels of 17,330 human genes across 9,783 samples from 175 types of healthy and pathological tissues. Genome Biol 2008, 9:R139.

43. Cheng LL, Lean CL, Bogdanova A, Wright Jr SC, Ackerman JL, Brady TJ, Garrido $L$ : Enhanced resolution of proton NMR spectra of malignant lymph nodes using magic-angle spinning. Magn Reson Med 1996, 36:653-658.

44. Moka D, Vorreuther R, Schicha H, Spraul M, Humpfer E, Lipinski M, Foxall PJD, Nicholson JK, Lindon JC: Biochemical classification of kidney carcinoma biopsy samples using magic-angle-spinning ${ }^{1} \mathrm{H}$ nuclear magnetic resonance spectroscopy. J Pharmaceut Biomed Anal 1998, 17:125-132.

45. Millis K, Weybright P, Campbell N, Fletcher JA, Fletcher CD, Cory DG, Singer S: Classification of human liposarcoma and lipoma using ex vivo proton NMR spectroscopy. Magn Reson Med 1999, 41:257-267.

46. Cheng LL, Burns MA, Taylor JL, He W, Halpern EF, McDougal WS, Wu CL: Metabolic characterization of human prostate cancer with tissue magnetic resonance spectroscopy. Cancer Res 2005, 65:3030-3034.

47. Sitter B, Sonnewald U, Spraul M, Fjösne HE, Gribbestad IS: High-resolution magic angle spinning MRS of breast cancer tissue. NMR Biomed 2002, 15:327-337.

48. Cheng LL, Chang IW, Smith BL, Gonzalez RG: Evaluating human breast ductal carcinomas with high-resolution magic-angle spinning proton magnetic resonance spectroscopy. J Magn Reson 1998, 135:194-202.

49. Marini C, lacconi C, Giannelli M, Cilotti A, Moretti M, Bartolozzi C: Quantitative diffusion-weighted MR imaging in the differential diagnosis of breast lesion. Eur Radiol 2007, 17:2646-2655.

50. Giskeødegård GF, Grinde MT, Sitter B, Axelson DE, Lundgren S, Fjøsne HE, Dahl S, Gribbestad IS, Bathen TF: Multivariate modeling and prediction of breast cancer prognostic factors using MR metabolomics. J Proteome Res 2010, 9:972-979.

51. Brochez V, Van Heuverswyn D, Diniz JA, De Potter C, Van den Eeckhout E:
Cellular uptake and retention measurements of alkylphosphocholines in the SK-BR-3 breast cancer and molt-4 leukemia cell line using capillary gas chromatography. Lipids 1999, 34:511-516.

52. Berzas JJ, Rodríguez J, Contento AM, Cabello MP: Determination of drugs used in advanced breast cancer by capillary gas chromatography of pharmaceutical formulations. J Sep Sci 2003, 26:908-914.

53. Daniel C, Gaskell S, Bishop H, Nicholson R: Determination of tamoxifen and an hydroxylated metabolite in plasma from patients with advanced breast cancer using gas chromatography-mass spectrometry. J Endocrino/ 1979 83:401-408.

54. Wolff MS, Toniolo PG, Lee EW, Rivera M, Dubin N: Blood levels of organochlorine residues and risk of breast cancer. J Nat Cancer Inst 1993, 85:648-652.

55. Ingram D, Sanders K, Kolybaba M, Lopez D: Case-control study of phytooestrogens and breast cancer. Lancet 1997, 350:990-994.

56. Nam H, Chung BC, Kim Y, Lee KY, Lee D: Combining tissue transcriptomics and urine metabolomics for breast cancer biomarker identification. Bioinformatics 2009, 25:3151-3157.

57. Woo HM, Kim KM, Choi MH, Jung BH, Lee J, Kong G, Nam SJ, Kim S, Bai SW, Chung $\mathrm{BC}$ : Mass spectrometry based metabolomic approaches in urinary biomarker study of women's cancers. Clin Chim Acta 2009, 400:63-69.

58. Kind T, Wohlgemuth G, Lee DY, LuY, Palazoglu M, Shahbaz S, Fiehn O: FiehnLib: mass spectral and retention index libraries for metabolomics based on quadrupole and time-of-flight gas chromatography/mass spectrometry. Anal Chem 2009, 81:10038-10048.

59. Kumari S, Stevens D, Kind T, Denkert C, Fiehn O: Applying in-silico retention index and mass spectra matching for identification of unknown metabolites in accurate mass GC-TOF mass spectrometry. Anal Chem 2011 , 83:5895-5902.

60. Abate S, Ahn YG, Kind T, Cataldi TRI, Fiehn O: Determination of elemental compositions by gas chromatography/time-of-flight mass spectrometry using chemical and electron ionization. Rapid Commun Mass Spectrom 2010, 24:1172-1180.

61. Schymanski E, Bataineh M, Goss KU, Brack W: Integrated analytical and computer tools for structure elucidation in effect-directed analysis. Trends Anal Chem 2009, 28:550-561.

62. Hummel J, Strehmel N, Selbig J, Walther D, Kopka J: Decision tree supported substructure prediction of metabolites from GC-MS profiles. Metabolomics 2010, 6:322-333.

63. Neumann S, Böcker S: Computational mass spectrometry for metabolomics: Identification of metabolites and small molecules. Analyt Bioanalyt Chem 2010, 398:2779-2788.

doi:10.1186/gm336

Cite this article as: Denkert $C$, et al: Metabolomics of human breast cancer: new approaches for tumor typing and biomarker discovery. Genome Medicine 2012, 4:37. 